\title{
Quality Controlled EMG Signal Compression using Linear and Non Linear Transforms
}

\author{
Vibha Aggarwal, Sandeep Gupta, Manjeet Singh Patterh, Lakhwinder Kaur
}

\begin{abstract}
In today's era of telemedicine, data and graphical records are required to be transmitted over noisy, power limited and band limited channels. The effective compression is the best alternate to save time and bandwidth. For Electromyogram (EMG) signal, that are huge in data size, must be compressed in such a way so that can be recovered with minimum alterations. This work focused on the tuneable method to compress EMG signals, with linear and non linear transforms. The analysis is based upon compression factor $(\mathrm{CF})$ and percentage root mean square difference (PRD). The results helps to conclude that non linear transform method have precedence over the linear transforms for almost entire range of user defined PRD (UPRD).
\end{abstract}

Keywords : Compression, EMG signals, Linear transform, Non Linear transform, Quality Control.

\section{INTRODUCTION}

Electromyogram (EMG) signals are due to the neuromuscular activities which makes the muscles contraction. This causes the generation of electrical currents which rely on the anatomical and physiological properties of muscles. EMG studies are used to help the diagnosis and management of disorders such as the muscular dystrophies and neuropathies. With the available EMG recorders, the collected data is huge in size as it is required to be measured for large period of time at multiple frequencies. If this data is used for storage or/and transmission needs without any signal processing, then it will require large space and bandwidth. Signal acquisition methods provide the solution to said hassle by representing the same graph with fewer bits (i.e. compression of signal). The compressed graphs used for any purpose can be recovered (i.e. decompression of signal) as original without any loss of signal quality. This will leads to efficient use of memory device as well as the channel bandwidth.

The compression of steady state and dynamic EMG signals with embedded zero tree wavelet and standard wavelet transform has been done by Norris et al. to access the difference between PRD and compression factor [20]. Researchers proposed different algorithms using linear

Revised Manuscript Received on October 10, 2019

* Correspondence Author

Vibha Aggarwal, College of Engineering and Management, Punjabi University Neighborhood Campus, Rampura Phul, Punjab, India. Email: vibha_ec@yahoo.co.in

Sandeep Gupta*, College of Engineering and Management, Punjabi University Neighborhood Campus, Rampura Phul, Punjab, India. Email: sandeeprple@pbi.ac.in

Manjeet Singh Patterh, University College of Engineering, Punjabi University, Patiala, Punjab, India. Email: pattarms@ieee.org

Lakhwinder Kaur, University College of Engineering, Punjabi University, Patiala, Punjab, India. Email: mahal2k8@yahoo.com transform for compression of EMG signals. Discrete wavelet transform (DWT) outperformed Discrete cosine transform (DCT) for EMG compression [6]. A new algorithm for compression of EMG signal using DWT was proposed, which was normalized and quantized using dynamic bit allocation scheme based on Kohonen neural network and then quantized coefficients were encoded by arithmetic encoder [5], [7]. The EMG signal when passed through the noisy channel may damage but can be recovered at receiving end with acceptable quality when processed during compression at transmitting side with DCT and DWT method [22]. Ntsama et al. use the JPEG 2000 to compress the EMG signal [23]. Later on, Ntsama et al. do the comparison of EMG signal compression by DCT and DWT using vector quantization, Set partitioning in hierarchical trees (SPIHT) and arithmetic coding [21]. Colince et al. find the trade-off between quality and CF when use the combination of Differential pulse-code modulation (DPCM) along with DCT and DWT [12].

Researchers also proposed different algorithms using non-linear transform for compression of EMG signals. Ele et al. presented modified lifting scheme for EMG signal compression to enhance the $\mathrm{CF}$ and to optimize the channel bandwidth [16].

EMG signal compression method was also proposed by using Autoregressive modelling theory [8]. If the shape of the signal waveform has to be preserved after compression, it will results in high compression factor $(\mathrm{CF})$. Further, Carotti et al [9], [10] preserved the shape of EMG signal waveform, results in reduction of CF by almost $9 \%$. Afterwards, Carotti et al. improve the quality of reconstruction of signal by exploiting both intra- and inter channel redundancy to achieve high $\mathrm{CF}$ without significant increase in signal-channel coding complexity [11].

EMG signals are segmented in two dimensional signals and managed to exploit the correlation property [13]-[15]. That signals are then encoded with JPEG2000 algorithm.

Form the literary support signal representation can be done by using appropriate transform method which are categorised as: linear transforms and nonlinear transforms. The linear transform segmented the signal in various parts based on its features by multiplication with transfer functions for example Discrete Cosine Transform (DCT), Discrete Wavelet Transform (DWT), Fractional Fourier Transform (FRFT) etc. Linear transforms has various features like progressive image transmission, ease of compressed signal manipulation, region of interest coding etc. 


\section{Quality Controlled EMG Signal Compression using Linear and Non Linear Transforms}

Linear transforms are non-adaptive methods so causes huge number of coefficients appears at discontinuities (Gibbs phenomenon). Whereas non linear methods (i,e Morphological Haar wavelet transform (MHAAR), Essentially non-oscillatory cell average transform (ENOCA), Lifting wavelet transform (LWT) are adaptive in nature so lessen the coefficients that occurs because of discontinuity in signal, resulting high compression ratio.

From the previous work, the quality controlled compression method with EMG signal is till date the untouched area and even most of the work was carried out with linear transform methods like DCT and DWT. Very few work has been reported by using non linear technique. However, there is no information available to compare the linear and non linear transforms for compression of EMG signals. This work introduced the linear FRFT and other non linear methods to compress the EMG signals. In this study, we also tried to justify the use of linear and non linear transform approach for EMG signals.

\section{METHODOLOGY}

For the proposed work, the data set was taken from Physionet database [19] to compare the three signal samples of 1-minute duration that belongs to a young healthy person of age 44 years, a middle age 57 year old person who is suffering from myopathy and old person of 62 year age and suffering from neuropathy.

In this research work, linear as well as non linear transform techniques are used to obtain the zero mean. To match the predefined user-specified percentage root mean square difference (UPRD), threshold values of coefficients (i.e maximum and minimum values) are obtained by using Bisection algorithm. Positioning mapping is done from the threshold values that are in the lookup tables. Huffman coding is used to encode the lookup table entries. Non-zero coefficient values are quantized using MaxLloyd quantizer, which is required to improve the signal to noise ratio. Arithmetic encoder is used to encode the quantized values [1]-[4].

The used transformation techniques operate at different level of decompositions. In ENOCA it is with one stage and two-degree interpolation. For DWT, EMG signal is decomposed to six levels using biorthogonal 4.4 filter, whereas for MHAAR and FRFT the level of decomposition is one and two respectively. LWT use the filter cdf 97.

Signal quality is percentage of root mean square difference (PRD) dependent, with the increase in PRD the quality of signal sullied, but it will also increase the compression factor (CF). Increased CF leads to optimize the memory and bandwidth which is the mandatory condition for valuable compression. With this proposed tunable algorithm user can achieve improved quality at cost of bandwidth and memory and on contrary side user can save bandwidth.

The CF and PRD are calculated as equation 1 and 2 respectively [21].

$$
C F=\frac{E M G_{\text {orig }}-E M G_{\text {Com }}}{E M G_{\text {orig }}} \times 100 \%
$$

Where $E M G_{\text {orig }}$ and $E M G_{\text {com }}$ are the original and compressed file lengths respectively.

$$
P R D=\frac{\sqrt{\sum_{n=1}^{k}\left(E M G_{\text {org }}[n]-E M G_{\text {rec }}[n]\right)^{2}}}{\sqrt{\sum_{n=1}^{k}\left(E M G_{\text {org }}[n]\right)^{2}}}
$$

Where $E M G_{\text {org }}[n]$ is the original signal and $E M G_{r e c}[n]$ is the reconstructed signal and $\mathrm{k}$ is the length of EMG signal.

Pictorial version of linear transform methods (DCT, DWT and FRFT) and non linear transforms (MHAAR, LWT and ENOCA) and for EMG signal and the comparison between original, reconstructed and error signal was done by selecting emg_healthym at UPRD=3.

\section{RESULT AND DISCUSSION}

This work computes the CF and PRD of compressed EMG signal that are obtained from three different age group persons with different medical history. The observations from simulated results inferred that non linear transform techniques provide high PRD and CF than linear transform methods.

From the table 1, it can be observed that for emg_healthym and emg_neuropathym signals, with non linear transform techniques, the achieved CF is higher for most of the PRD values. For emg_healthym UPRD 1,2,3,4 and 5, MHAAR CF is 89.7, DWT 91.27, DWT 92.81, ENOCA 94.90 and LWT 94.63 respectively. For emg_neuropathym UPRD 1,2,3,4 and 5 DWT CF is 89.17, ENOCA 91.49, LWT 91.78, ENOCA 92.12 and ENOCA 92.45 respectively. For emg_myopathym UPRD 1,2,3,4 and 5, LWT CF is 87.40, LWT 87.40, LWT 89.81, DWT 90.11 and DWT 90.82 respectively. For emg_myopathym signal the CF is higher with linear DWT technique but if compared with non linear LWT results, the difference in $\mathrm{CF}$ value is non-significant. Hence it can be concluded that non linear has the precedence over the linear transform technique.

Table 2 support the improvement of proposed technique on the bases of CF and PRD. A lot of work has been done in past but when compared with present proposed techniques then it can be inferred that from Norris et. al [20] to Ntsama et. al [21] the PRD was calculated for fixed CF that ranges from 70 to 85. In this work, $\mathrm{CF}$ is calculated for fixed PRD. It is because PRD reflects the quality of the signal and user can tune the PRD as per particular requirements. To the best of our knowledge till date, the researchers achieved PRD from 0.20 to 15.76 , that is why the range for PRD taken in this work confined to 1 to 5 which is adequate range for acceptable quality of signal. Most of the researchers use the wavelet transform in their work and same transform when used in presented work show the supremacy in results. The work when extended for other liner and non linear transforms, then obtained results are further optimized.

Pictorially the original, reconstructed and error signals are shown in Figures 1-6. These figures show that the reconstructed signal is matching with the original signal. 


\section{CONCLUSION}

The tuneable EMG signal compression is the grey area for researchers and this work focused on the untouched liner and non linear transforms. The results were compared with the previous work in which the obtained $\mathrm{CF}$ was 85 with $P R D=1.45$. In this work at $U P R D=1$, the achieved $C F$ is 89.72 by using MHAAR transform and when UPRD increased to 5, it boost the CF to 94.84 with ENOCA transform. The presented work, when compared among various proposed transforms and with literature work concludes that non linear transforms have better CF for various UPRD. This work can be further extended with other techniques to optimize the said parameters for particular environment.

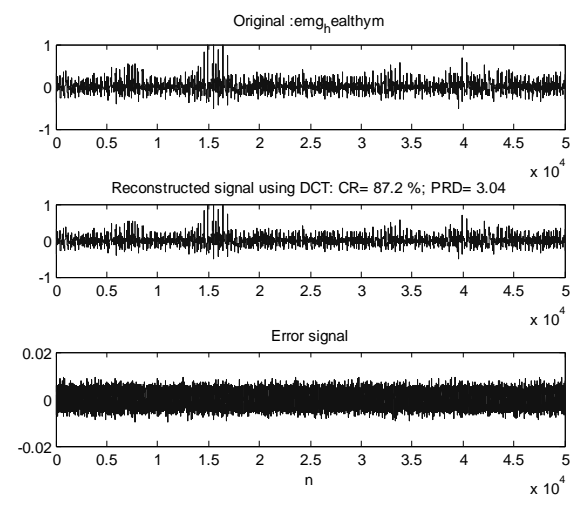

Fig. 1.Compression waveform of record emg_healthy using DCT at UPRD=3.

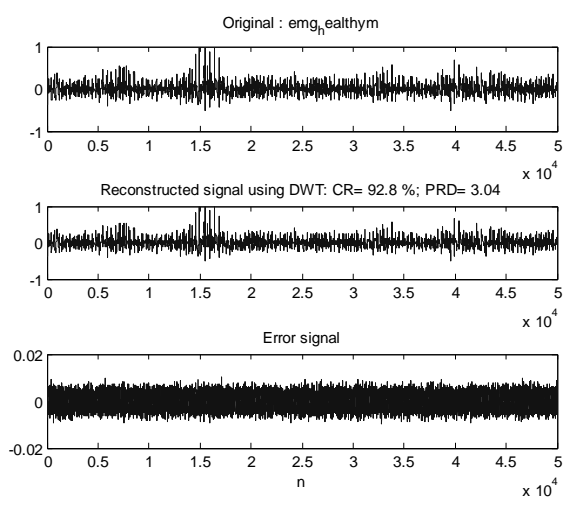

Fig. 2.Compression waveform of record emg_ healthy using DWT at UPRD=3.

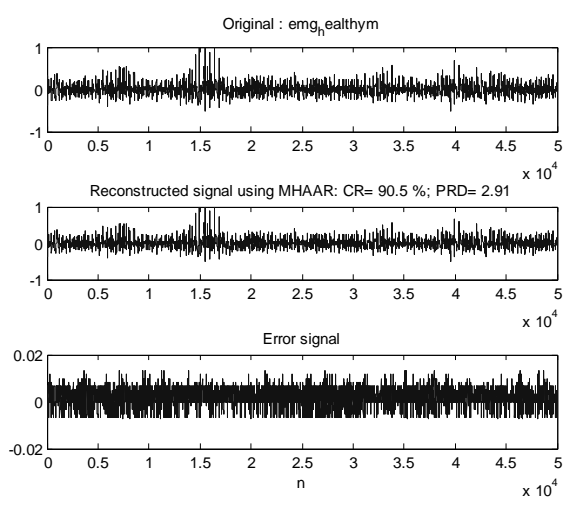

Fig. 3.Compression waveform of record emg_ healthy using MHAAR at UPRD=3.

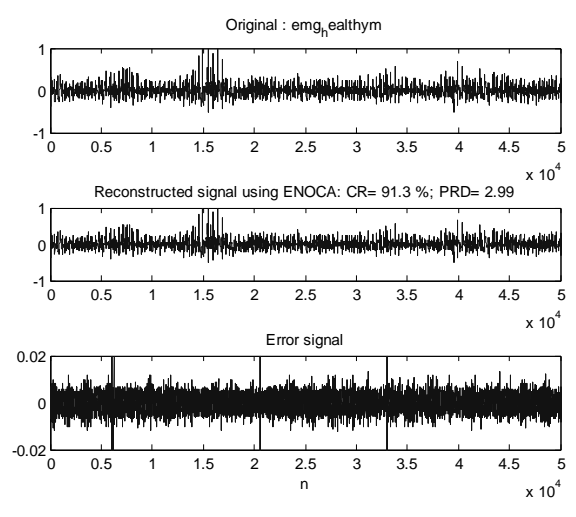

Fig. 4.Compression waveform of record emg_ healthy using ENOCA at $\mathrm{UPRD}=3$.

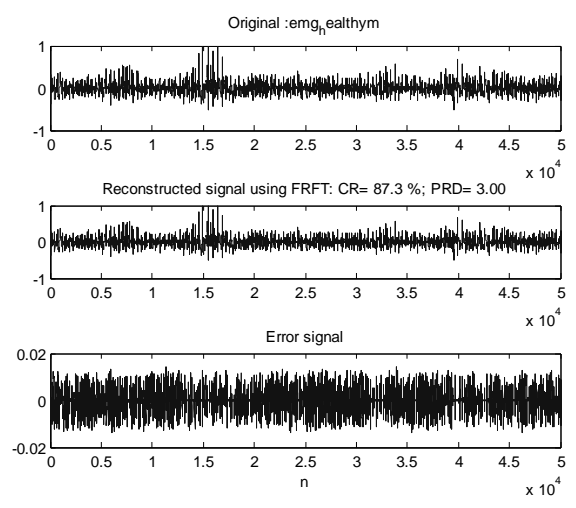

Fig. 5.Compression waveform of record emg_ healthy using FRFT at UPRD=3.

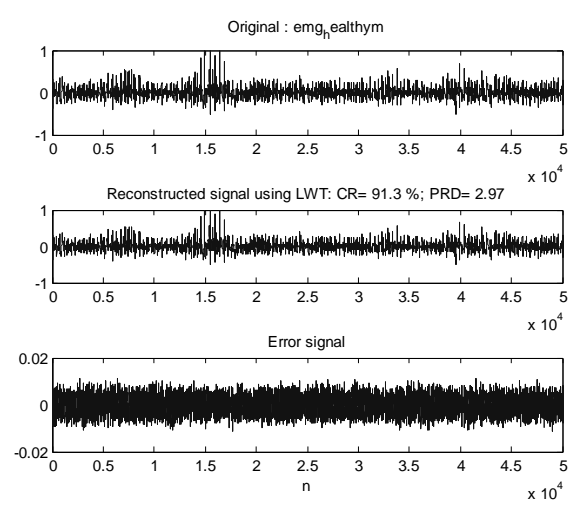

Fig. 6.Compression waveform of record emg_ healthy using $\mathrm{LWT}$ at $\mathrm{UPRD}=3$. 


\section{Quality Controlled EMG Signal Compression using Linear and Non Linear Transforms}

Table- I: Performance of Linear and Non Linear Transforms For EMG Signal

\begin{tabular}{|c|c|c|c|c|c|c|c|c|c|c|c|c|c|c|c|}
\hline \multicolumn{16}{|c|}{ Duration of the signal 1 minute, Quantization bits $=12$ bits } \\
\hline \multicolumn{16}{|c|}{ emg_myopathym } \\
\hline \multirow{2}{*}{ Transform } & \multicolumn{3}{|c|}{$\mathrm{UPRD}^{\mathrm{a}}=1$} & \multicolumn{3}{|c|}{$\mathrm{UPRD}^{\mathrm{a}}=2$} & \multicolumn{3}{|c|}{$\mathrm{UPRD}^{\mathrm{a}}=3$} & \multicolumn{3}{|c|}{$\mathrm{UPRD}^{\mathrm{a}}=4$} & \multicolumn{3}{|c|}{$\mathrm{UPRD}^{1}=5$} \\
\hline & $\mathrm{BPRD}^{\mathrm{b}}$ & $\mathrm{QPRD}^{\mathrm{c}}$ & $\mathrm{CF}^{\mathrm{d}}$ & $\mathrm{BPRD}^{\mathrm{b}}$ & $\mathrm{QPRD}^{\mathrm{c}}$ & $\mathrm{CF}^{\mathrm{d}}$ & $\mathrm{BPRD}^{\mathrm{b}}$ & QPRD $^{c}$ & $\mathrm{CF}^{\mathrm{d}}$ & $\mathrm{BPRD}^{\mathrm{b}}$ & $\mathrm{QPRD}^{\mathrm{c}}$ & $\mathrm{CF}^{\mathrm{d}}$ & $\mathrm{BPRD}^{2}$ & $\mathrm{QPRD}^{3}$ & $\mathrm{CF}^{4}$ \\
\hline DCT & 0.99 & 1.00 & 87.11 & 1.99 & 1.98 & 87.11 & 3.02 & 3.03 & 87.12 & 3.96 & 3.96 & 87.12 & 4.98 & 4.98 & 86.12 \\
\hline DWT & 1.00 & 1.03 & 86.98 & 1.99 & 2.02 & 86.98 & 2.98 & 2.99 & 89.42 & 4.01 & 4.03 & 90.12 & 5.02 & 5.03 & 90.82 \\
\hline MHAAR & 0.99 & 0.99 & 87.16 & 2.09 & 2.08 & 87.16 & 3.13 & 2.96 & 89.72 & 3.97 & 3.96 & 89.76 & 4.81 & 4.81 & 89.84 \\
\hline ENOCA & 1.00 & 1.19 & 87.40 & 1.97 & 2.11 & 87.40 & 2.98 & 2.99 & 89.80 & 3.97 & 3.99 & 89.77 & 4.95 & 4.97 & 89.77 \\
\hline FRFT & 1.00 & 1.01 & 87.10 & 1.99 & 1.99 & 87.11 & 2.98 & 2.99 & 87.11 & 4.00 & 4.02 & 87.11 & 4.96 & 4.96 & 87.11 \\
\hline LWT & 0.99 & 0.99 & 87.40 & 1.98 & 2.00 & 87.40 & 2.99 & 2.98 & 89.81 & 4.00 & 3.98 & 89.70 & 5.00 & 5.01 & 89.65 \\
\hline \multicolumn{16}{|c|}{ emg_neuropathym } \\
\hline DCT & 0.99 & 1.01 & 79.68 & 2.01 & 2.01 & 79.75 & 3.00 & 3.01 & 79.83 & 4.03 & 4.03 & 79.89 & 5.02 & 5.02 & 79.95 \\
\hline DWT & 1.00 & 1.07 & 89.17 & 1.99 & 2.02 & 89.75 & 2.98 & 2.99 & 90.14 & 3.97 & 3.98 & 90.33 & 5.00 & 5.01 & 90.54 \\
\hline MHAAR & 1.00 & 1.02 & 86.80 & 2.01 & 2.04 & 90.48 & 3.03 & 3.04 & 90.92 & 4.02 & 4.01 & 91.28 & 5.04 & 5.04 & 91.61 \\
\hline ENOCA & 0.99 & 1.00 & 88.17 & 1.99 & 2.01 & 91.49 & 3.01 & 3.04 & 91.71 & 4.00 & 3.97 & 92.12 & 4.95 & 4.95 & 92.45 \\
\hline FRFT & 0.99 & 1.01 & 82.96 & 1.98 & 1.99 & 84.74 & 3.00 & 3.01 & 86.93 & 4.04 & 4.04 & 90.72 & 5.02 & 5.01 & 91.04 \\
\hline LWT & & & & 2.00 & 2.01 & 91.41 & 3.02 & 3.02 & 91.78 & 4.00 & 4.00 & 92.08 & 5.01 & 5.02 & 92.31 \\
\hline
\end{tabular}

Table- II: Comparison of The results (CF, PRD) For EMG Signal with Literature work

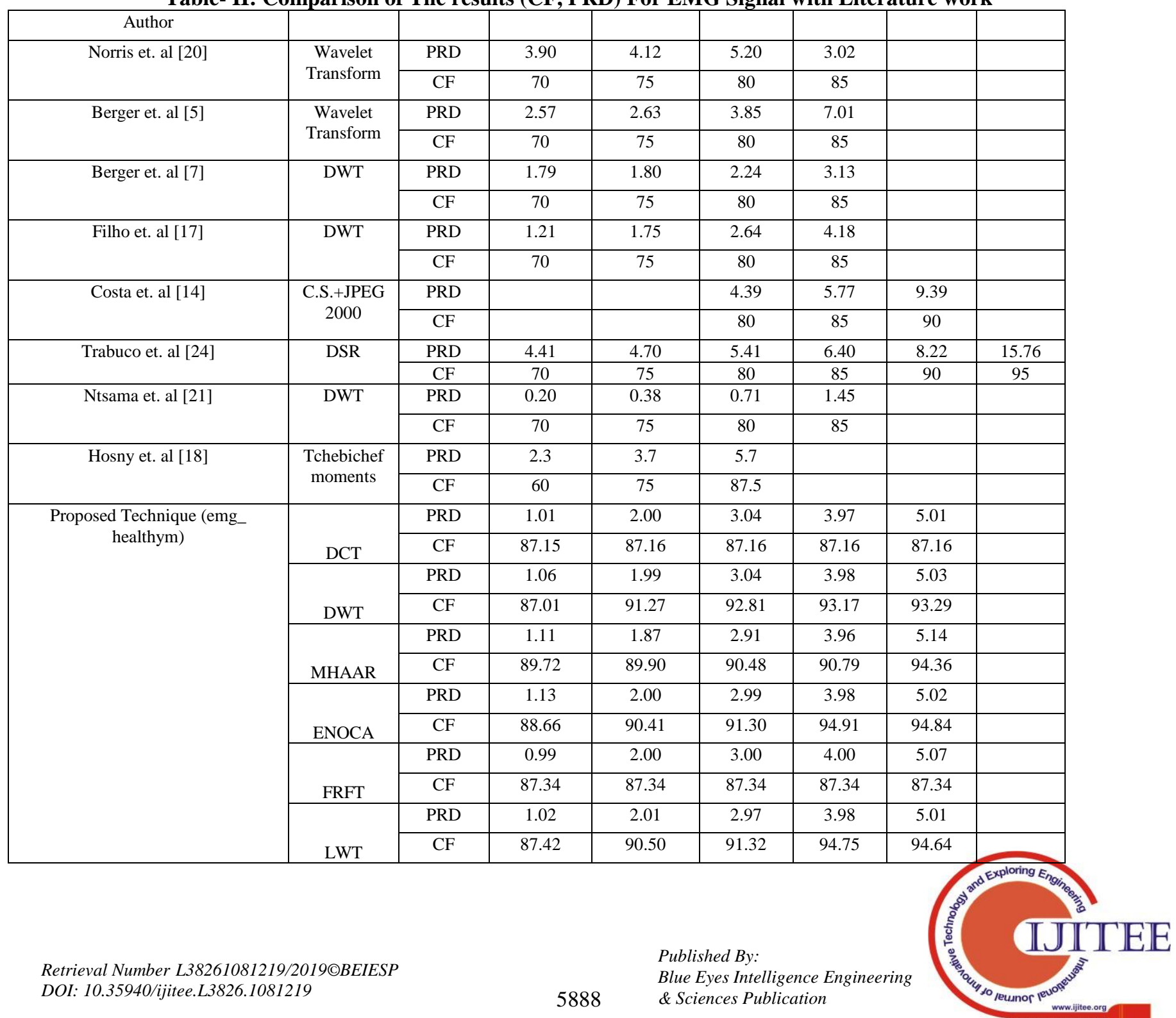




\section{REFERENCES}

1. V. Aggarwal, and M. S. Patterh, "Performance Analysis of Non-Linear Transformational ECG Compression Method". in Biomedical Engineering: Applications, Basis and Communications (BME), vol. 21, 2009, pp. 245-251.

2. V. Aggarwal, and M. S. Patterh, "Quality controlled ECG compression using essentially non-oscillatory point-value decomposition (ENOPV) technique" in Digital Signal Processing, vol. 22, 2012, pp. 878-884.

3. V. Aggarwal, and M. S. Patterh, "Quality Controlled ECG Compression using Slantlet Transform and Lifting Wavelet transform with and without Normalization", in International Journal of Electronics, vol. 100, 2013, pp. 626-636.

4. V. Aggarwal, M. S. Patterh, and V. K. Singla, "Comparative studies of discrete cosine transform and lifting wavelet transform techniques for compression of blood pressure signal in salt sensitive Dahl rat", in International Journal Computational Systems Engineering, vol. 4 (2/3), 2018, pp. 165-170.

5. P. A. Berger , F. A. O. Nascimento, J. C. Carmo, and A. F. Rocha, "Compression of EMG signals with wavelet transform and artificial neural networks" in Physiol Meas, vol. 27 (6), 2006, pp. 457-465.

6. P. A. Berger , F. A. O. Nascimento, J. C. Carmo, A. F. Rocha and I. Dos Santos, "Algorithm for compression of EMG signals", in Proceedings of the 25th Annual International Conference of the IEEE Engineering in Medicine Biology Society, 2003, pp. 17-21.

7. P. A. Berger, F. A. O. Nascimento, A. F. Rocha, and J. L. A. Carvalho, "A new wavelet-based algorithm for compression of EMG signals", in Proceedings of the 29th annual international conference of the IEEE engineering in medicine and biology society, Lyon, 2007, pp. 1554-1557.

8. E. S. G. Carotti, J. C. De Martin, D. Farina, and R. Merletti, "Linear predictive coding of myoelectric signals", in Acoustics, speech and signal processing, ICASSP'05, IEEE international conference, 2005 pp. 629-632.

9. E. S. G. Carotti, J. C. De Martin, R. Merletti, and D. Farina "Compression of surface EMG Signals with Algebric Code Excited linear Prediction", in ICASSP'06, IEEE International conference, 2006, pp. 1148-1151.

10. E. S. G. Carotti, J. C. De Martin, R. Merletti, and D. Farina, "Compression of surface EMG signals with algebraic code excited linear prediction", in Med Eng. Phys., vol. 29, issue 2, 2007, pp. 253-258.

11. E. S. G. Carotti, J. C. De Martin, R. Merletti, and D. Farina, "Compression of multidimensional biomedical signals with spatial and temporal codebook-excited linear prediction", in IEEE Trans. Biomed. Eng., vol. 56 (11), 2009, pp. 2604-2610.

12. W. Colince, E. P. Ntsama, and P. Ele, "Exploitation of Differential pulse Code Modulation for Compression of EMG Signals by Combination of DWT and DCT", in American Journal of Biomedical Engineering, vol. 4(2), 2014, pp. 25-32.

13. M. V. C. Costa, P. A. Berger, A. F. Da Rocha, J. L. A. Carvalho, and F A. O. Nascimento, "Compression of Electromyographic Signals Using Image Compression Techniques", in Proceedings of the 30th Annual International Conference of the IEEE Engineering in Medicine and Biology Society, Vancouver, 2008, pp. 2948-2951.

14. M. V. C. Costa, J. L. A. Carvalho, P. A. Berger, A. F. Da Rocha, and F. A. O. Nascimento, "Compression of surface electromyographic signals using two-dimensional techniques", in Recent Advances in Biomedical Engineering. Edited by: Naik GR. Rijeka, Croatia: In Tech, 2009, pp. 17-38.

15. M. V. C. Costa, J. L. A. Carvalho, P. A. Berger, A. Zaghetto, A. F. Da Rocha, and F. A. O. Nascimento, "Two-dimensional compression of surface electromyographic signals using column-correlation sotrting and image encoders", in Proceedings of the 31st annual international conference of the IEEE engineering in medicine and biology society, Minneapolis, 2009, pp. 428-431.

16. P. Ele, E. P. Ntsama, and E. Tonye, "Characterization of Modified Lifting Scheme Cpmpression method Of EMG Signal", in Journal of Communication and Computer, vol. 8, 2011, pp. 1013-1021.

17. E. B. Filho, Da Silva, and M. B. Carvalho, "On EMG signal compression with recurrent patterns", in IEEE Trans. Biomed. Eng., vol. 55 (7), 2008, pp. 1920-1923.

18. K. M. Hosny, A. K. Mohamed, and E. R. Mohamed, "Efficient Compression of Bio Signal by Using Tchebichef Moments and Artificial Bee Colony", in Biocybernetics Biomedical Engineering, 2018

19. https://physionet.org/physiobank/database/emgdb/
20. J. A. Norris, K. Englehart, and D. Lovely, "Steady-state and dynamic myoelectric signal compression using embedded zero-tree wavelets", in Proceedings of the 23rd annual international conference of the IEEE engineering in medicine biology society, Istanbul, Turkey, vol. 2, 2001, pp. 1879-1882.

21. E. P. Ntsama, W. Colince, and P. Ele, "Compression Study of EMG signals Compression by Methods Transforms Using Vector Quantization, SPIHT and Arithmetic Coding”, in Springer Plus, vol, 5(1), 2016, pp. 1-18.

22. E. P. Ntsama, P. Ele, and I. B. Kabiena, " Compression approach of EMG signal using 2D discrete wavelet and cosine transforms”, in Am. J Signal Process, vol. 3(1), 2013, pp. 10-16.

23. E. P. Ntsama, E. S. Z. D. Pierre, Z. D. Serfebé ZD, and T. Emmanuel, "Evaluation of EMG signals compression by JPEG 2000 called 1D", in Int. J. Eng. Technol. (IJET), vol. 5(1), 2013, pp. 44-51.

24. M. H. Trabuco, M. V. C. Costa, and A. O. N. Francisco, "S-EMG signal compression based on domain transformation and spectral shape dynamic bit allocation", in Biomed. Eng. Online, 2014, pp.13-22.

\section{AUTHORS PROFILE}

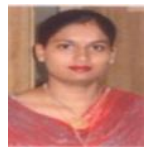

Vibha Aggarwal earned her Bachelor and Maste degrees in Electronics and Communication Engineering. She obtained her PhD from Punjabi University Patiala. She has 12-years of Teaching and professional experience. Currently, she is working as an Assistant Professor (Electronics and Communication Engineering) in College of Engineering and Management (CoEM). She has total of 22 research international and national publications in journals and conferences. Her main research area is digital signal processing. She is a member of professional bodies ISTE and Punjab Academy of Sciences.

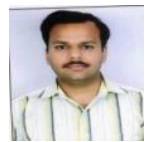

Sandeep Gupta received his Bachelor and Master degrees in Electronics and Communication Engineering in 2001 and 2007 respectively. He received his $\mathrm{PhD}$ from GKU, Talwandi Sabo (PB). Presently he is working as Assistant Professor at CoEM, Punjabi University Neighbourhood campus Rampura Phul. His research interests include wireless communication and MANETs. He is life time member of professional bodies ISTE and Punjab Academy of Sciences.

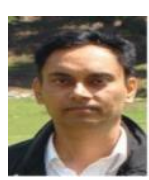

Manjeet Singh Patterh received his Bachelor's degree from the Madhav Institute of Technology and Science (MITS), Gwalior, MP, India and Master's degree from the Birla Institute of Technology and Science (BITS), Pilani, both in Electronics Engineering. He received his $\mathrm{PhD}$ degree from the Punjab Technical University, Jalandhar. He has 60 publications in international, national refereed journals and conferences. He is having over 23 years of teaching experience. He is currently working as a Professor in the Department of Electronics and Communication Engineering at the Punjabi University, Patiala. His current interests are digital signal processing, wireless communication systems and networking. He is a member of IEEE and life member of ISTE, IE(I) and IETE.

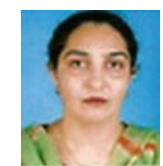

Lakhwinder Kaur is a Professor in Computer Engineering at Punjabi University, Patiala, India. She received her $\mathrm{PhD}$ from PTU, Jalandhar, India. She has been in teaching since September 1992. Her research interes includes image processing and wireless networks. She has published more than 50 research papers in International Journals, Conference proceedings as well as books. 